\title{
The Metabolism and Distribution of Docosapentaenoic Acid (n-6) in the Liver and Testis of Growing Rats
}

\author{
Phyllis S. Y. Tam, ${ }^{1}$ Rumi Sawada, ${ }^{2}$ Yan Cui, ${ }^{1}$ Akiyo Matsumoto, ${ }^{3}$ and Yoko Fujiwara ${ }^{1, \dagger}$ \\ ${ }^{1}$ Graduate School of Humanities and Sciences, Ochanomizu University, \\ 2-1-1 Otsuka, Bunkyo-ku, Tokyo 112-8610, Japan \\ ${ }^{2}$ Division of Medical Devices, National Institute of Health Sciences, \\ 1-18-1 Kamiyoga, Setagaya-ku, Tokyo 158-8501, Japan \\ ${ }^{3}$ Department of Clinical Dietetics and Human Nutrition, Josai University, \\ 1-1 Keyakidai, Sakado, Saitama 350-0295, Japan
}

Received April 14, 2008; Accepted July 2, 2008; Online Publication, October 7, 2008

[doi:10.1271/bbb.80249]

To investigate the metabolism and distribution of docosapentaenoic acid (22:5n-6, DPA) in the liver and testis of growing rats, 22:5n-6 was administered to their dams. Newborn rats with a low hepatic arachidonic acid (20:4n-6, AA) level were generated by administrating a diet rich in docosahexaenoic acid (22:6n-3, DHA) but n-6 fatty acid (FA) free to pregnant dams. After parturition, 22:5n-6 or linoleic acid (18:2n-6, LA) was administered with a high level of 22:6n-3 to the dams until weaning. At weaning, the hepatic 20:4n-6 level was significantly highest in the DPA-DHA but not LA-DHA diet-fed animals. The hepatic delta-6 desaturase (D6D) mRNA abundance was significantly lower in both the LA-DHA and DPA-DHA diet-fed animals, connoted with the 20:4n-6 content recovered by $22: 5 n-6$ that did not involve D6D and supporting the occurrence of retroconversion in the liver of the growing rats. The low D6D level in the 3-week-old testis was not in proportion to the elevated 22:5n-6 level, implying that early testicular 22:5n-6 accumulation might require supply from the circulation system.

Key words: docosapentaenoic acid; docosahexaenoic acid; arachidonic acid; delta-6 desaturase; retroconversion

Docosahexaenoic acid (22:6n-3, DHA), the most abundant n-3 fatty acid normally present in human and animal tissues, has specific functions in the brain and retina. $^{1-4)}$ It has been reported that infant formula containing 22:6n-3 was associated with improved growth and development. ${ }^{5,6)}$ Moreover, formula containing 22:6n-3 has been shown to enable preterm infants to achieve immune development similar to that seen with breast-milk feeding. ${ }^{7)}$ Arachidonic acid (20:4n-6, AA), which is important in cell membrane phospholipids and serves as the precursor of eicosanoids, is maintained at a constant level under normal conditions in vivo. It has been shown that breast milk containing 20:4n-6 contributed to infant development. ${ }^{8)}$ There is also report that 20:4n-6 deficiency in preterm infants may lead to impaired growth over the first year of life.9) In studies on 22:6n-3 supplementation, however, a high dietary intake of 22:6n-3 resulted not only in an increased 22:6n-3 content, but also in a drastically decreased 20:4n-6 content. ${ }^{10,11)}$ This decrease in $20: 4 n-6$ has been proposed to be related to the inhibition of delta- 6 desaturase (D6D), reducing the synthesis of 20:4n-6 from linoleic acid (18:2n-6, LA). Both 22:6n-3 and 20:4n-6 are essential components for infant growth and development, and a lack of either may lead to impaired growth. Therefore, the 20:4n-6 decline caused by $22: 6 n-3$ still requires a solution.

The docosapentaenoic acid (22:5n-6, DPA) content in most organisms is low, the n-3 isomer of 22:5n-6 in most fish oils being at a higher level than the $n-6$ isomer. ${ }^{12)}$ It is also known that $22: 5 \mathrm{n}-6$ is $\beta$-oxidized to $20: 4 n-6,{ }^{13-16)}$ and that a deficiency of $n-3$ essential fatty acids in animals causes a compensatory rise in the $22: 5 n-6$ level in the brain/retina, ${ }^{17,18)}$ although the physiological function of $22: 5 n-6$ has not yet been clarified. In our previous in vivo and in vitro study, $22: 5 n-6$ was found to blunt the decrease in $20: 4 n-6$ level, but 18:2n-6 could not prevent the same decline in 20:4n- 6 caused by a high $22: 6 n-3$ administration level, implying that the $20: 4 n-6$ level increased by $22: 5 n-6$ supplementation was not affected by $22: 6 n-3 .{ }^{19}$ )

There has been no previous report on the effect of $22: 6 n-3$ on the 20:4n-6 level from pregnancy; studies on

$\dagger$ To whom correspondence should be addressed. Fax: +81-3-5978-5751; E-mail: fujiwara.yoko@ocha.ac.jp

Abbreviations: D6D, delta-6 desaturase; D5D, delta-5 desaturase; FA, fatty acid; PUFA, polyunsaturated fatty acid; GC, gas-chromatography 
the effect of $22: 5 n-6$ and $18: 2 n-6$ on the biosynthesis of 20:4n-6 conditioned by high $22: 6 n-3$ supplementation are also limited. It would therefore be valuable to compare the effects of $22: 5 n-6$ and $18: 2 n-6$, the two possible sources of 20:4n-6 accumulation, under a high 22:6n-3 administration level on the accumulation of 20:4n-6 in pups from pregnancy to weaning.

A second objective would be to clarify the source of the testicular accumulation of 22:5n-6 during growth, because it has been found that 22:5n-6 was present in a high concentration specifically in rat testis, where the level of 22:5n-6 has been found to be independent of the kind and quantity of oil in the diet. ${ }^{19)}$ Therefore, the testicular 22:5n-6 accumulation was investigated in newborn and weaned rats to clarify the source of $22: 5 n-6$ in the testis during growth.

\section{Materials and Methods}

Materials. The DPA-DHA oil prepared from a singlecell marine microbe was presented by Suntory (Osaka, Japan). Safflower oil, rapeseed oil and soybean oil were presented by The Nissin Oillio Group (Tokyo, Japan). DHA-35G oil was presented by Japan Scientific Feeds Association. All other chemicals and reagents were of analytical grade.

Animals and diets. All procedures for the use and care of the animals for laboratory research were approved by the Animal Experiment Ethics Committee of Ochanomizu University. Eleven-week-old Wistar rats were purchased from Sankyo Labo Service Corporation (Tokyo, Japan). All the rats were initially fed on a commercial diet (CE-2) from Nippon Clea Co. (Tokyo, Japan) for one week. The animals were housed individually in an air-conditioned room at $23 \pm 1{ }^{\circ} \mathrm{C}$ with a $12 \mathrm{~h}$ light-dark cycle and were given the experimental diets and water ad libitum.

The test schedule was shown in Fig. 1. After coitus, the dams were fed with DHA-rich oil (DHA group: n-6 FA-free diet) or safflower oil (SO group: n-6 FA-rich control diet) until parturition to generate newborn rats with low hepatic 20:4n-6 content. At birth, the dams were subdivided into the LA-DHA group (LA, 26\%) and DPA-DHA group (LA, 17\%; DPA, 7.8\%) with no difference in body weight (data not shown). Before breast feeding, newborn pups with similar body weight were randomly selected from the DPA-DHA and LA-DHA groups to provide 5-10 samples for an analysis of the FA composition and D6D mRNA expression level at birth (week 0). The dams in the SO group continued to receive the same diet. The newborn pups were breast-fed for 3 weeks until weaning.

All the diets were prepared by mixing $5 \%(\mathrm{w} / \mathrm{w})$ of the experimental oil mixtures with the basal diet. The basal diet, which was prepared according to the standards given by the American Institute of Nutrition $(\mathrm{AIN}),{ }^{20)}$ was provided by Eisai Co., and contained the

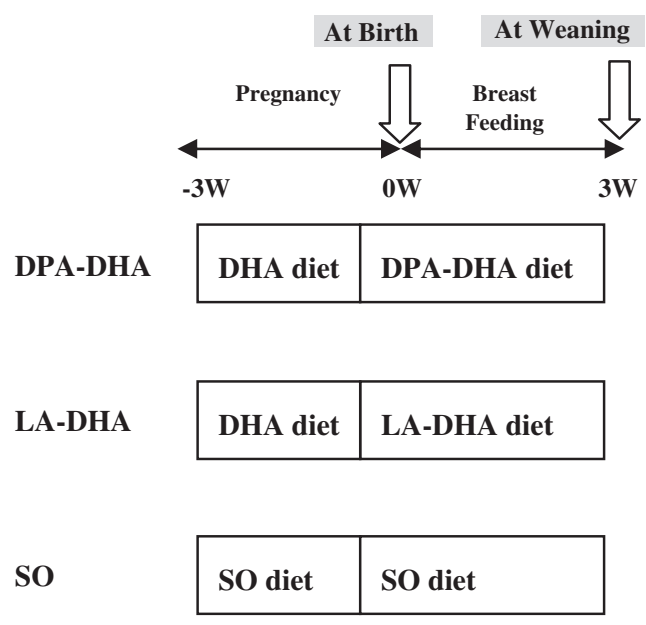

Fig. 1. Experimental Design and Diets Given to the Dams.

SO, safflower oil diet; LA-DHA, 18:2n-6-rich diet; DPA-DHA, 22:5n-6-rich diet; $-3 \mathrm{~W}$, three weeks before parturition; $0 \mathrm{~W}$, at parturition; $3 \mathrm{~W}$, at weaning. All the experimental diets were administered to dams. Analyses were conducted at the birth (week 0) and weaning (week 3) stages.

Table 1. Fatty Acid Composition of the Diets (\%)

\begin{tabular}{lrrrc}
\hline \hline & SO & DHA & LA-DHA & DPA-DHA \\
\hline $16: 0$ & 8.8 & 20.1 & 7.0 & 40.7 \\
$18: 0$ & 3.3 & 4.4 & 2.9 & 2.2 \\
$18: 1(\mathrm{n}-9)$ & 16.2 & 21.2 & 31.9 & 4.4 \\
$18: 2(\mathrm{n}-6)$ & 70.9 & 3.0 & 26.0 & 17.3 \\
$20: 4(\mathrm{n}-6)$ & 0.0 & 0.0 & 0.0 & 0.0 \\
$22: 5(\mathrm{n}-6)$ & 0.0 & 0.0 & 0.0 & 7.8 \\
$18: 3(\mathrm{n}-3)$ & 0.9 & 0.0 & 5.4 & 0.0 \\
$20: 5(\mathrm{n}-3)$ & 0.0 & 10.8 & 0.0 & 0.0 \\
$22: 6(\mathrm{n}-3)$ & 0.0 & 40.4 & 26.9 & 28.8 \\
\hline
\end{tabular}

Each Value is expressed as a percentage of weight. SO, safflower oil diet; DHA, DHA-rich diet; LA-DHA, 18:2n-6-rich diet; DPA-DHA, 22:5n-6-rich diet.

following percentage of ingredients according to weight: casein, 20; glucose, 25; sucrose, 25; cornstarch, 15; filter paper, 5; mineral mixture (AIN-76), 3.5; vitamin mixture (AIN-76), 1; choline bitartrate, 0.2; and DLmethionine, 0.3. The oil mixtures were prepared as follows: (i) SO diet, safflower oil; (ii) DHA diet, DHA$35 \mathrm{G}$ oil; (iii) DPA-DHA diet, DPA-DHA oil/safflower oil, 4:1; (iv) LA-DHA diet, (Soybean oil/rapeseed oil, 1:1)/DHA ethyl ester(65:35). The total amounts of n-6 and n-3 fatty acids in the dams' diets were adjusted to similar levels (Table 1).

Tissues collection. All animals were killed by blood collection from the abdominal aorta. Collected tissues were washed by saline and stored at $-80^{\circ} \mathrm{C}$.

Lipid analyses. Lipids were extracted from tissues of the rats by the method of Folch et al. ${ }^{21)}$ Each lipid extract was methyl esterified with $\mathrm{HCl} /$ methanol by the method mentioned in our previous paper to measure the composition of fatty acids in the tissue. ${ }^{22)}$ The fatty acid 
methyl esters were measured by gas-liquid chromatography (Perkin Elmer AutoSystemGC; Palo Alto, CA, USA) with a Rascot Silliar 5CP capillary column $(0.25 \mathrm{~mm} \times 50 \mathrm{~m}$; Nihon Chromato Works, Tokyo, Japan) under the conditions previously described in detail. $^{22)}$

Real-time PCR analysis. Total RNA was extracted from the tissues by the acid guanidinium-phenol-chloroform method. ${ }^{23)}$ The mRNA expression level of D6D was determined by the real time PCR method.

The Primer design of D6D was based on the sequences obtained from GenBank and the location of the open reading frame as determined with TaqMan Applied Biosystems Primer Express 1.0 software. The forward and reverse primers used were as follows: (5'-GTTCTTCTTTCTCCTCCTGTCCC-3') and (5'CATTGCCGAAGTACGAGAGGAT-3'). Each sample was subjected to quantitative real-time PCR, using the GeneAmp 5700 sequence detection system (Applied Biosystems).

Statistical analysis. Each result is shown as the mean \pm SD. The significance of differences was evaluated by analysis of variance (ANOVA) and the Bonferroni-Dunn post-hoc test, or by Student's $t$-test. Analyses were performed by using the StatView (System 4.02) computer package or Microsoft Excel software.

\section{Results}

\section{Growth parameters and tissue weights}

No significant differences in the food intake and body weight gain were apparent among the animal groups (data not shown). We conclude that the administration of the various dietary PUFAs had no adverse influence on the growth of the rats.

\section{Effect on the fatty acid composition in the liver of pups}

Pregnant dams were fed by the DHA-rich diet for three weeks until parturition, and the hepatic fatty acid composition of the pups was investigated at birth (week 0) (Table 2). The 22:6n-3 content in the DHA diet was incorporated into the newborn liver of the DHA group feeding dams with the DHA diet containing only $3 \%$ of $18: 2 n-6$ as the n- 6 fatty acid successfully in created a newborn liver with a significantly low level of 20:4n-6 compared to the SO group. The other major $n-6$ FAs were at a very low level $(18: 2 n-6)$ or not detected $(22: 4 n-6$ and $22: 5 n-6)$ in the newborn liver of the DHA group.

The low 20:4n-6 environment created at birth provided an appropriate platform to compare the efficiency of $18: 2 n-6$ and $22: 5 n-6$, the two possible sources of 20:4n-6 accumulation. 18:2n-6 and 22:5n-6 were respectively supplemented with $22: 6 n-3$ in the diet for the
Table 2. Fatty Acid Composition in the Liver at Birth (Week 0)

\begin{tabular}{lcc}
\hline \hline & SO & DHA \\
\hline $18: 0$ & $15.1 \pm 0.47^{\mathrm{a}}$ & $12.8 \pm 0.23^{\mathrm{b}}$ \\
$18: 1 \mathrm{n}-9$ & $5.87 \pm 0.63$ & $4.76 \pm 0.37$ \\
$18: 2 \mathrm{n}-6$ & $13.2 \pm 1.25^{\mathrm{a}}$ & $2.70 \pm 0.13^{\mathrm{b}}$ \\
$20: 4 \mathrm{n}-6$ & $13.6 \pm 0.71^{\mathrm{a}}$ & $4.99 \pm 0.29^{\mathrm{b}}$ \\
$22: 4 \mathrm{n}-6$ & $1.78 \pm 0.11$ & $\mathrm{ND}$ \\
$22: 5 \mathrm{n}-6$ & $2.45 \pm 0.14$ & ND \\
$20: 5 \mathrm{n}-3$ & $\mathrm{ND}$ & $0.90 \pm 0.17$ \\
$22: 6 \mathrm{n}-3$ & $4.99 \pm 0.34^{\mathrm{a}}$ & $7.88 \pm 0.38^{\mathrm{b}}$ \\
\hline
\end{tabular}

Dams were fed on the experimental diets for 3 weeks until parturition. The FA composition in newborn liver was analyzed by GC. Each result is expressed in $\mathrm{nmol} / \mathrm{g}$ of tissue by the mean $\pm \mathrm{SD}(\mathrm{n}=5-10)$. SO, safflower oil diet; DHA, DHA-rich diet. The significance of differences between different dietary treatments was analyzed by Student's t-test. Bars not sharing a common roman letter are significantly different $(\mathrm{p}<0.05)$.

Table 3. Fatty Acid Composition of the Milk (\%)

\begin{tabular}{lrrc}
\hline \hline & SO & LA-DHA & DPA-DHA \\
\hline $16: 0$ & 20.7 & 22.7 & 24.3 \\
$18: 0$ & 2.9 & 2.7 & 3.9 \\
$18: 1(\mathrm{n}-9)$ & 13.1 & 12.4 & 9.8 \\
$18: 2(\mathrm{n}-6)$ & 17.0 & 6.7 & 6.4 \\
$20: 4(\mathrm{n}-6)$ & 0.6 & 0.0 & 0.4 \\
$22: 5(\mathrm{n}-6)$ & 0.0 & 0.0 & 1.2 \\
$18: 3(\mathrm{n}-3)$ & 0.2 & 1.1 & 0.0 \\
$20: 5(\mathrm{n}-3)$ & 0.0 & 0.3 & 0.0 \\
$22: 6(\mathrm{n}-3)$ & 0.0 & 2.8 & 6.1 \\
\hline
\end{tabular}

Each value is expressed as a percentage of weight. SO, safflower oil diet; LA-DHA, 18:2n-6-rich diet; DPA-DHA, 22:5n-6-rich diet.

dams to feed the LA-DHA and DPA-DHA groups. To confirm whether the various dietary PUFAs had been incorporated into the milk of the dams in each group, the milk was collected at weaning (week 3 ) for a fatty acid analysis (Table 3). 18:2n-6, 22:5n-6 and 22:6n-3, which were the major PUFAs in the dams' diet, were found to have been incorporated into the milk. The fatty acid profile in the liver at weaning (Week 3) is shown in Table 4. Although fed by dams whose diet had been supplemented with a high 22:6n-3 content, 18:2n-6 and $22: 5 n-6$ was respectively incorporated into the liver of the LA-DHA and DPA-DHA pups. The 18:2n-6 level was significantly higher in the LA-DHA group, and the 22:5n-6 level was significantly higher in the DPA-DHA group. The level of 20:4n-6 in the liver of the weaned DPA-DHA group was significantly higher than that of the LA-DHA group. Comparing the 20:4n-6 levels in the livers of growing rats in the LA-DHA and DPA-DHA groups, 22:5n-6 was found to be more effective in its contribution to $20: 4 n-6$ accumulation when administered with a significant amount of 22:6n-3.

Effect on the D6D mRNA expression level in the liver of the pups

$\mathrm{D} 6 \mathrm{D}$, the rate-determining enzyme in both the $\mathrm{n}-3$ and n-6 pathway, was investigated in the liver of the newborn rats (week 0) (Fig. 2). The mRNA expression level of D6D was significantly low in the DHA group 
Table 4. Fatty Acid Composition in the Liver at Weaning (Week 3)

\begin{tabular}{lccc}
\hline \hline & SO & LA-DHA & DPA-DHA \\
\hline $18: 0$ & $16.7 \pm 0.92$ & $17.4 \pm 0.37$ & $16.8 \pm 0.42$ \\
$18: 1 \mathrm{n}-9$ & $6.87 \pm 0.78^{\mathrm{a}}$ & $4.55 \pm 0.24^{\mathrm{b}}$ & $4.04 \pm 0.19^{\mathrm{b}}$ \\
$18: 2 \mathrm{n}-6$ & $18.7 \pm 1.88^{\mathrm{a}}$ & $7.69 \pm 0.17^{\mathrm{b}}$ & $5.93 \pm 0.21^{\mathrm{b}}$ \\
$20: 4 \mathrm{n}-6$ & $16.8 \pm 1.08^{\mathrm{a}}$ & $5.74 \pm 0.21^{\mathrm{b}}$ & $8.44 \pm 0.45^{\mathrm{c}}$ \\
$22: 4 \mathrm{n}-6$ & $1.73 \pm 0.16$ & ND & ND \\
$22: 5 \mathrm{n}-6$ & $2.93 \pm 0.14^{\mathrm{a}}$ & ND & $2.09 \pm 0.20^{\mathrm{b}}$ \\
$20: 5 \mathrm{n}-3$ & ND & $2.20 \pm 0.12^{\mathrm{a}}$ & $0.83 \pm 0.09^{\mathrm{b}}$ \\
$22: 6 \mathrm{n}-3$ & $3.84 \pm 0.30^{\mathrm{a}}$ & $22.8 \pm 1.68^{\mathrm{b}}$ & $22.8 \pm 1.43^{\mathrm{b}}$ \\
\hline
\end{tabular}

Dams were fed on the experimental diets for 3 weeks until weaning. The FA composition in the weaned liver was analyzed by GC. Each Result is expressed in $\mathrm{nmol} / \mathrm{g}$ of tissue by the mean $\pm \mathrm{SD}(\mathrm{n}=3-6)$. SO, safflower oil diet; LA-DHA, 18:2n-6-rich diet; DPA-DHA, 22:5n-6-rich diet. The significance of differences between different dietary treatments was evaluated by an analysis of variance (ANOVA) and the Bonferroni-Dunn post-hoc test. Bars not sharing a common roman letter are significantly different $(\mathrm{p}<0.05)$.

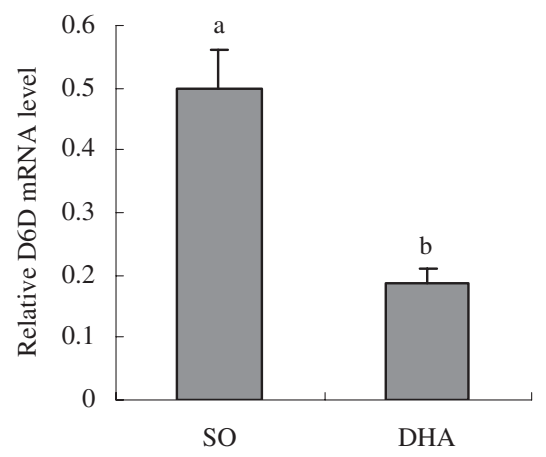

Fig. 2. Effect on D6D in the Liver of Newborn Rats (Week 0).

The mRNA expression level of D6D was measured by real-time PCR. Each result is expressed as the relative concentration $(\mathrm{n}=5-10)$. SO, safflower oil diet; DHA, 22:6n-3-rich diet. The significance of differences between different dietary treatments was analyzed by Student's t-test. Bars not sharing a common roman letter are significantly different $(\mathrm{p}<0.05)$.

liver. The D6D mRNA abundance in the weaned rats at week 3 is shown in Fig. 3. The D6D mRNA levels were very low in both the LA-DHA and DPA-DHA group weaned liver, there being no significant difference between the D6D mRNA levels in both these groups.

\section{Effect on the fatty acid composition in the testis of the pups}

The fatty acid composition in newborn testis at week 0 is shown in Table 5. 22:6n-3, which was the major PUFA included in the DHA diet, was found to be significantly high in DHA group testis. No significant difference was found between the levels of $20: 4 n-6$ and 22:5n-6, the major n-6 metabolites in the testis.

The testicular fatty acid composition at week 3 is shown in Table 6. 22:6n-3 was significantly high in the testis of the weaned LA-DHA and DPA-DHA groups, reflecting its effect from the milk of the dams fed with LA-DHA or DPA-DHA diets. 22:5n-6 in the testis of the weaned DPA-DHA group was significantly higher than that of the LA-DHA group.

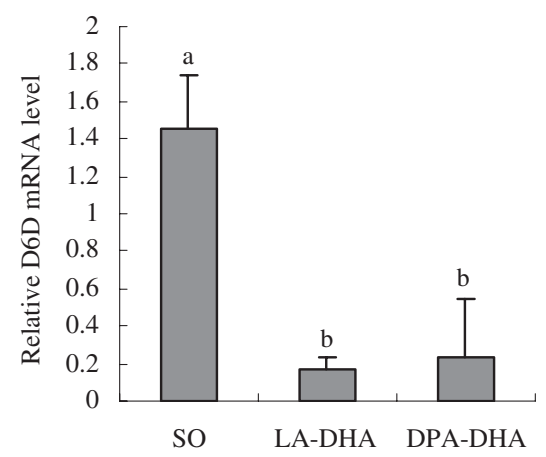

Fig. 3. Effect on D6D in the Liver of Weaned Rats (Week 3).

The mRNA expression level of D6D was measured by real-time PCR. Each result is expressed as the relative concentration $(\mathrm{n}=3-6)$. SO, safflower oil diet; LA-DHA, 18:2n-6-rich diet; DPA-DHA, 22:5n-6-rich diet. The significance of differences between different dietary treatments was evaluated by an analysis of variance (ANOVA) and the Bonferroni-Dunn post-hoc test. Bars not sharing a common roman letter are significantly different $(\mathrm{p}<0.05)$.

Table 5. The Fatty Acid Composition in the Testis at Birth (Week 0)

\begin{tabular}{lcc}
\hline \hline & SO & DHA \\
\hline $18: 0$ & $10.1 \pm 1.97^{\mathrm{a}}$ & $17.5 \pm 2.54^{\mathrm{b}}$ \\
$18: 1 \mathrm{n}-9$ & $4.13 \pm 0.27$ & $5.65 \pm 1.10$ \\
$18: 2 \mathrm{n}-6$ & $1.32 \pm 0.19^{\mathrm{a}}$ & $2.19 \pm 0.40^{\mathrm{b}}$ \\
$20: 4 \mathrm{n}-6$ & $2.93 \pm 0.23$ & $2.59 \pm 0.21$ \\
$22: 4 \mathrm{n}-6$ & $0.61 \pm 0.02$ & ND \\
$22: 5 \mathrm{n}-6$ & $0.34 \pm 0.01$ & $0.51 \pm 0.10$ \\
$20: 5 \mathrm{n}-3$ & ND & $0.37 \pm 0.09$ \\
$22: 6 \mathrm{n}-3$ & $0.65 \pm 0.03^{\mathrm{a}}$ & $1.78 \pm 0.02^{\mathrm{b}}$ \\
\hline
\end{tabular}

Dams were fed on the experimental diets for 3 weeks until parturition. The FA composition in the newborn testis was analyzed by GC. Each result is expressed in $\mathrm{nmol} / \mathrm{g}$ of tissue by the mean $\pm \mathrm{SD}(\mathrm{n}=5-10)$. SO, safflower oil diet; DHA, 22:6n-3-rich diet. The significance of differences between different dietary treatments was analyzed by Student's t-test. Bars not sharing a common roman letter are significantly different $(\mathrm{p}<0.05)$.

Table 6. Fatty Acid Composition in the Testis at Weaning (Week 3)

\begin{tabular}{lccc}
\hline \hline & SO & LA-DHA & DPA-DHA \\
\hline $18: 0$ & $5.43 \pm 0.08$ & $5.05 \pm 0.31$ & $5.61 \pm 0.19$ \\
$18: 1 \mathrm{n}-9$ & $4.85 \pm 0.10^{\mathrm{a}}$ & $5.94 \pm 0.51^{\mathrm{b}}$ & $4.40 \pm 0.23^{\mathrm{a}}$ \\
$18: 2 \mathrm{n}-6$ & $1.40 \pm 0.04^{\mathrm{a}}$ & $1.49 \pm 0.15^{\mathrm{a}}$ & $0.95 \pm 0.05^{\mathrm{b}}$ \\
$20: 4 \mathrm{n}-6$ & $5.45 \pm 0.22^{\mathrm{a}}$ & $3.32 \pm 0.39^{\mathrm{b}}$ & $3.52 \pm 0.25^{\mathrm{b}}$ \\
$22: 4 \mathrm{n}-6$ & $0.78 \pm 0.02^{\mathrm{a}}$ & $0.19 \pm 0.03^{\mathrm{b}}$ & $0.26 \pm 0.02^{\mathrm{b}}$ \\
$22: 5 \mathrm{n}-6$ & $3.27 \pm 0.26^{\mathrm{a}}$ & $0.18 \pm 0.02^{\mathrm{b}}$ & $0.63 \pm 0.07^{\mathrm{c}}$ \\
$20: 5 \mathrm{n}-3$ & ND & $0.26 \pm 0.03^{\mathrm{a}}$ & $0.11 \pm 0.01^{\mathrm{b}}$ \\
$22: 6 \mathrm{n}-3$ & $0.64 \pm 0.02^{\mathrm{a}}$ & $3.24 \pm 0.47^{\mathrm{b}}$ & $2.56 \pm 0.24^{\mathrm{b}}$ \\
\hline
\end{tabular}

Dams were fed on the experimental diets for 3 weeks until weaning. The FA composition in weaned testis was analyzed by GC. Each result is expressed in $\mathrm{nmol} / \mathrm{g}$ of tissue by the mean $\pm \mathrm{SD}(\mathrm{n}=3-6)$. SO, safflower oil diet; LADHA, 18:2n-6-rich diet; DPA-DHA, 22:5n-6-rich diet. The significance of differences between different dietary treatments was evaluated by an analysis of variance (ANOVA) and the Bonferroni-Dunn post-hoc test. Bars not sharing a common roman letter are significantly different $(\mathrm{p}<0.05)$.

Effect on the D6D mRNA expression level in the testis of the pups

The testicular D6D mRNA expression could not be investigated at week 0 due to the limited size of the 


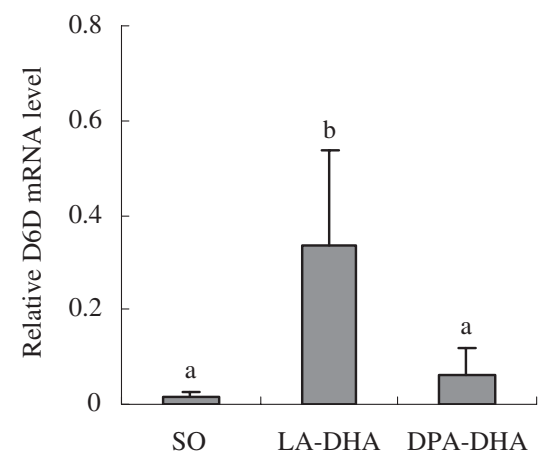

Fig. 4. Effect on D6D in the Testis of Weaned Rats (Week 3). The mRNA expression level of D6D was measured by real-time PCR. Each Result is expressed as the relative concentration $(\mathrm{n}=3-6)$. SO, safflower oil diet; LA-DHA, 18:2n-6-rich diet; DPA-DHA, 22:5n-6-rich diet. The significance of differences between different dietary treatments was evaluated by an analysis of variance (ANOVA) and the Bonferroni-Dunn post-hoc test. Bars not sharing a common roman letter are significantly different $(\mathrm{p}<0.05)$

newborn organs. The results for the testis of the weaned pups at week 3 are shown in Fig. 4. The D6D mRNA level was significantly higher in the testis of the LADHA group than of the DPA-DHA group. There was no significant difference between the D6D mRNA level in the DPA-DHA and SO groups. Focusing on the results for the SO group, the low D6D expression level was not in proportion to the increased accumulation of major $n-6$ metabolites, especially $22: 5 n-6$, in the testis of the SO group.

\section{Discussion}

Feeding pregnant dams with a n-6 FA-free diet in the present study successfully created newborn pups with a significantly low level of 20:4n-6 in the liver of the DHA group which provided a favorable environment to compare the effect of the two possible sources for 20:4n-6 synthesis: $18: 2 n-6$ and 22:5n-6. After 3 weeks of diet intervention with the dams fed the LA-DHA diet, the 20:4n-6 level in their pups was unchanged from the 20:4n-6 level at birth (Tables 3 and 4). However, intervention with 22:5n-6 in the dams fed with the DPA-DHA diet resulted in the hepatic 20:4n-6 level in their pups being increased by 1.5 -fold, showing that $20: 4 n-6$ synthesis by $22: 5 n-6$ was more efficient than by $18: 2 n-6$. Moreover, DHA diet intervention in the dams resulted in the decline of D6D mRNA expression in both the newborn and weaned liver of their pups (Figs. 2 and 3). This D6D decline did not stop the increase of 20:4n-6 by $22: 5 n-6$, which implies that the conversion of 22:5n-6 to 20:4n-6 did not involve D6D and supports the retroconversion of $22: 5 n-6$ to $20: 4 n-6$ that had been reported in our previous study. ${ }^{19)}$ Studies on the retroconversion of $22: 5 n-6$ to $20: 4 n-6$ in rat liver have reported that the retroconversion occured with $22: 4 n-6$ as the intermediate, suggesting that retroconversion took place by direct hydrogenation and subsequent $\beta$-oxidation. ${ }^{24)}$

It has been well reported that a 20:4n-6 decline would be caused by $22: 6 n-3$ administration in vivo. ${ }^{25-28)}$ In the present study, the hepatic 20:4n-6 decline in newborn rats resulted from indirect diet intervention by supplementing the experimental diets fed to the dams. The D6D mRNA expression level showed that D6D was significantly low in the newborn pups fed by the dams that had received the DHA diet, which may account for the decline of 20:4n-6 in the newborn liver. As D6D is the major enzyme catalyzing the rate-determining first step of the n-6 and n-3 PUFA metabolic pathway, which involves the conversion of linoleic acid (LA, 18:2n-6) to $\gamma$-linolenic acid (GLA, 18:3n-6) and of ALA to 18:4n-3, the metabolism of 18:2n-6 cannot start without D6D. There have been studies reporting the inhibitory effect of PUFAs on the expression of D5D and D6D in the liver through the regulation of sterol-regulatory element binding protein 1 (SREBP-1) at the transcription level. ${ }^{29,30)}$ Moreover, it has been reported that the expression level of D5D and D6D was positively regulated by SREBP-1 in the liver as a result of the presence of a sterol-regulatory element in the promoter region of $\mathrm{D} 6 \mathrm{D}$ and probably $\mathrm{D} 5 \mathrm{D} .^{31,32)}$ According to these reports, the low D6D expression level caused by PUFAs in this study was probably related to the suppression of SREBP-1 at the transcription level.

$20: 4 n-6$ is an essential component of early growth. ${ }^{8,9)}$ Therefore, the abnormal decline of $20: 4 n-6$ caused by high 22:6n-3 supplementation still requires a solution. Our former and present studies provided animal in vivo and in vitro data demonstrating that the retroconversion of $22: 5 n-6$ to $20: 4 n-6$ was independent of a high $22: 6 n-3$ administration; this may suggest a solution to blunt the abnormal 20:4n-6 decline caused by a high 22:6n-3 administration by applying 22:5n-6 to $22: 6 n-3$. A human clinical study has reported the effect on cardiovascular risk factors of a marine oil rich in 22:6n-3 and 22:5n-6, similar to the oil used in this study, showing that the oil was safe and did not adversely affect cardiovascular disease. ${ }^{33)}$ In future, human data on the retroconversion of $22: 5 n-6$ to $20: 4 n-6$ caused by a high $22: 6 n-3$ administration will be required.

In our previous study, a high 22:5n-6 concentration was unique to the rat testis, where the level of 22:5n-6 was found to be independent of the quality and quantity of oil supplemented to the diet for mature rats. ${ }^{19)}$ The testis is an organ that consumes C22 PUFA to transport the sperm into the vesiculae seminalis. ${ }^{34)}$ At homeostasis, 22:5n-6 as the dominant C22 PUFA in the testis is believed to be involved in the function of sperm transportation. ${ }^{34)}$ A study on the testicular development of rats maintained from weaning age to 63 weeks has reported that rats fed with a n-6 FA deficient diet suffered testicular degeneration with the loss of germinal cells and the 22:5n-6 level was being markedly low in the testis. ${ }^{35)}$ In this study, the fatty acid profile in the testis of newborn and weaned rats showed that 22:5n-6 
in the testes was at a level independent of the dietary effect. These results support the unique presence and importance of 22:5n- 6 during the development of this organ (Tables 5 and 6).

It is known that testicular lipids have an active metabolism, arising from both dietary sources and from the processes of synthesis, elongation, desaturation, interconversion, esterification and oxidation by the testicular tissue itself, ${ }^{36)}$ although the source of $22: 5 n-6$ accumulation during early growth has not yet been clarified. In the present study, focusing on the results for the SO group testis at week 3, the low D6D expression level was not in proportion to the increased accumulation of major n-6 metabolites, especially 22:5n-6 (Table 6). This suggests that the accumulation of 22:5n-6 in the testis at the growing stage was not only supported by local production, but also by uptake from the circulation system, which was probably provided by synthesis in the liver. According to an analysis of mouse transcriptome, SREBP-1 was highly expressed in the liver, while its expression in the testis is less than 25\%. ${ }^{37)}$ Moreover, the same relative SREBP-1 expression in these tissues has been reported in rodent and human tissue. ${ }^{38)}$ The low testicular expression of the transcription factors may explain the lower desaturase response observed in the testis than in the liver.

In conclusion, the results of the present study demonstrate that the conversion of $22: 5 n-6$ to $20: 4 n-6$ was more effective than of $18: 2 n-6$ to $20: 4 n-6$ under the condition of a high $22: 6 n-3$ administration. A second finding in this study is the possibility of 22:5n-6 uptake from the circulation system for testicular 22:5n-6 accumulation during early growth.

\section{References}

1) Nuringer, M., Conner, W. E., Petten, C. V., and Barstad, L., Dietary omega-3 fatty acid deficiency and visual loss in infant rhesus monkeys. J. Clin. Invest., 73, 272-276 (1984).

2) Bazan, N. G., Reddy, R. S., Bazan, H. E. P., and Birkle, D. L., Metabolism of arachidonic and docosahexaenoic acids in the retina. Prog. Lipid Res., 25, 595-606 (1986).

3) Uauy, R. D., Birch, D. G., Tyson, J. E., and Hoffman, D. R., Effect of dietary omega-3 fatty acids on retinal function of very-low-birth-weight neonates. Pediatr. Res., 28, 485-492 (1990).

4) Sandra, N., Gharib, A., Croset, M., Moliere, P., and Lagarde, M., Fatty acid composition of the rat pineal gland. Dietary modifications. Biochim. Biophys. Acta, 1081, 75-78 (1991).

5) Koletzko, B., Decso, T., and Demmelmair, H., Arachidonic acid supply and metabolism in human infants born at full term. Lipids, 31, 79-83 (1996).

6) Makrides, B., Neumann, M., Simmer, K., Pater, J., and Gibson, R., Are long-chain polyunsaturated fatty acids essential nutrients in infancy? Lancet, 345, 1463-1468 (1995).

7) Field, C. J., Thomson, C. A., Van Aerde, J. E., Parrott, A., Euler, A., Lien, E., and Clandinin, M. T., Lower proportion of CD45RO + cells and deficient interleukin10 production by formula fed infants, compared with human-fed, is corrected with supplementation of longchain polyunsaturated fatty acids. J. Pediatr. Gastroenterol. Nutr., 31, 291-299 (2000).

8) Sanjurjo, P., Rodriguez-Alarcon, J., and RodriguezSoriano, J., Plasma fatty acid composition during the first week of life following feeding with human milk or formula. Acta Paediatr. Scand., 77, 202-209 (1988).

9) Carlson, S. E., Werkman, S. H., Peeples, J. M., Cooke, R. J., and Tolly, E. A., Arachidonic acid status correlates with first year growth in preterm infants. Proc. Natl. Acad. Sci. USA, 90, 1073-1077 (1993).

10) Cao, J. M., Blond, J. P., Juaneda, P., Durand, G., and Bezard, J., Effect of low levels of dietary fish oils on fatty acid desaturation and tissue fatty acids in obese and lean rats. Lipids, 30, 825-832 (1995).

11) Suarez, A., Faus, M. J., and Gil, A., Dietary long-chain polyunsaturated fatty acids modify heart, kidney, and lung fatty acid composition in weanling rats. Lipids, $\mathbf{3 1}$, 345-348 (1996).

12) Gunstone, F. D., Harwood, J. L., and Padley, F. B., "The Lipid Handbook," Chapman \& Hall, London, pp. 173175 (1994).

13) Verdino, B., Blank, M. L., Privett, O. S., and Lundberg, W. O., Metabolism of 4,7,10,13,16-docosapentaenoic acid in the essential fatty acid-deficient rat. J. Nutr., 83, 234-236 (1964).

14) Schlenk, H., Gellerman, J. L., and Sand, D. M., Retroconversion of polyunsaturated fatty acids in vivo by partial degradation and hydrogenation. Biochim. Biophys. Acta, 137, 420-425 (1967).

15) Kunau, W. H., Uber dei synthese der an allen doppelbindungen tritiummarkierten 4.7.10.13.16-docosapentaensaure und ihre umwandlung in 5.8.11.14-eicosatetraensaure bei fettfrei ernharten ratten. Z. Physiol. Chem., 349, 333-336 (1968).

16) Bridges, R. B., and Coniglio, J. G., The metabolism of $4,7,10,13,16-\left[5-{ }^{14} \mathrm{C}\right]$ docosapentaenoic acid in the testis of the rat. Biochim. Biophys. Acta, 218, 29-35 (1970).

17) Homayoun, P. G., Durand, G., Pascal, G., and Bourre, J. M., Alternation of fatty acid composition of adult rat brain capillaries and choroid plexus induced by a diet deficient in n-3 fatty acids: slow recovery after substitution with a non-deficient diet. J. Neurochem., 51, 45-48 (1988).

18) Guesnet, P., Pasval, G., and Durand, G., Effects of dietary alpha-linolenic acid deficiency during pregnancy and lactation on lipid fatty acid composition of liver and serum in the rat. Reprod. Nutr. Dev., 28, 275-292 (1988).

19) Tam, P. S., Umeda-Sawada, R., Yaguchi, T., Akimoto, K., Kiso, T., and Igarashi, O., The metabolism and distribution of docosapentaenoic acid (n-6) in rats and rat hepatocytes. Lipids, 35, 71-75 (2000).

20) American Institute of Nutrition, Report of the American Institute of Nutrition ad hoc committee on standards for nutritional studies. J. Nutr., 107, 1340-1348 (1977).

21) Folch, J., Lees, M., and Sloane-Stanley, G. H., A simple method for the isolation and purification of total lipids from animal tissues. J. Biol. Chem., 266, 497-509 (1957).

22) Fujiyama-Fujiwara, Y., Ohmori, C., and Igarashi, O., Metabolism of $\gamma$-linolenic acid in primary cultures of rat 
hepatocytes and in HepG2 cells. J. Nutr. Sci. Vitaminol., 35, 591-611 (1989).

23) Chomczynski, P., and Sacchi, N., Single-step method of RNA isolation by acid guanidinium thiocyanate-PhOHchloroform extraction. Anal. Biochem., 162, 156-159 (1987).

24) Kunau, W. H., Partial degradation of 4.7.10.13.16docosapentaenoic acid in rat liver. FEBS Lett., 16, 54 56 (1971).

25) Raimondi, L., Lodovici, M., Visioli, F., Sartiani, L., Cioni, L., Alfarano, C., Banchelli, G., Pirisino, R., Cecchi, E., Cerbai, E., and Mugelli, A., N-3 polyunsaturated fatty acids supplementation decreases asymmetric dimethyl arginine and arachidonate accumulation in aging spontaneously hypertensive rats. Eur. J. Nutr., 44, 327-333 (2005).

26) Wang, J. Y., and Saito, M., Dietary supplementation of n-3 fatty acids and hydroperoxide levels in rat retinas. Free Radic. Res., 35, 367-375 (2001).

27) Engler, M. M., Bellenger-Germain, S. H., Engler, M. B., Narce, M. M., and Poisson, J. P., Dietary docosahexaenoic acid affects stearic acid desaturation in spontaneously hypertensive rats. Lipids, 35, 1011-1015 (2000).

28) Engler, M. M., Engler, M. B., Kroetz, D. L., Boswell, K. D., Neeley, E., and Krassner, S. M., The effects of a diet rich in docosahexaenoic acid on organ and vascular fatty acid composition in spontaneously hypertensive rats. Prostaglandins Leukot. Essent. Fatty Acids, 61, 289-295 (1999).

29) Matsuzaka, T., Shimano, H., Yahagi, N., AmemiyaKudo, M., Yoshikawa, T., Hasty, A. H., Tamura, Y., Osuga, J., Okazaki, H., Iizuka, Y., Takahashi, A., Sone, H., Gotoda, T., Ishibashi, S., and Yamada, N., Dual regulation of mouse delta-5 and delta- 6 desaturase gene expression by SREBP-1 and PPAR $\alpha$. J. Lipid Res., 43, 107-114 (2002).

30) Horton, J. D., Goldstein, J. L., and Brown, M. S.,
SREBPs: activators of the complete program of cholesterol and fatty acid synthesis in the liver. J. Clin. Invest., 109, 1125-1131 (2002).

31) Xu, J., Teran-Garcia, M., Park, J. H., Nakamura, M. T., and Clarke, S. D., Polyunsaturated fatty acids suppress hepatic sterol regulatory element-binding protein-1 expression by accelerating transcript decay. J. Biol. Chem., 276, 9800-9807 (2001).

32) Xu, J., Cho, H., O'Malley, S., Park, J. H., and Clarke, S. D., Dietary polyunsaturated fats regulate rat liver sterol regulatory element binding proteins- 1 and -2 in three distinct stages and by different mechanisms. J. Nutr., 132, 3333-3339 (2002).

33) Sanders, T. A., Gleason, K., Griffin, B., and Miller, G. J., Influence of an algal triacylglycerol containing docosahexaenoic acid (22:6n-3) and docosapentaenoic acid (22:5n-6) on cardiovascular risk factors in healthy men and women. Br. J. Nutr., 95, 525-531 (2006).

34) Coniglio, J. G., Testicular lipids. Prog. Lipid Res., 33, 387-401 (1994).

35) Leat, W. M. F., Northrop, C. A., Harrison, F. A., and Cox, R. W., Effect of dietary linoleic and linolenic acids on testicular development in the rat. J. Exp. Physiol., 68, 221-231 (1983).

36) Coniglio, J. G., "The Testis" Vol. IV, eds. Johnson, A. D., and Gomes, W. R., Academic Press, New York, pp. 425-449 (1977).

37) Su, A. I., Cooke, M. P., Ching, K. A., Hakak, Y., Walker, J. R., Wiltshire, T., Orth, A. P., Vega, R. G., Sapinoso, L. M., Moqrich, A., Patapoutian, A., Hampton, G. M., Schultz, P. G., and Hogenesch, J. B., Large-scale analysis of the human and mouse transcriptomes. Proc. Natl. Acad. Sci., 99, 4465-4470 (2002).

38) Shimomura, I., Shimano, H., Horton, J. D., Goldstein, J. L., and Brown, M. S., Differential expression of exons 1a and $1 \mathrm{c}$ in mRNAs for sterol regulatory element binding protein-1 in human and mouse organs and cultured cells. J. Clin. Invest., 99, 838-845 (1997). 